\title{
IDENTIDAD NACIONAL Y SU RELACIÓN CON LA DOMINANCIA SOCIAL Y LA TOLERANCIA A LA TRANSGRESIÓN EN RESIDENTES DE BUENOS AIRES (ARGENTINA)
}

\section{NATIONAL IDENTITY AND ITS RELATIONSHIP WITH SOCIAL DOMINANCE AND TOLERANCE TRANSGRESSION IN BUENOS AIRES' RESIDENTS (ARGENTINA)}

\author{
Santiago Monsegur ${ }^{*}$, Agustín Espinosa $^{* *}$ y Maite Beramendi ${ }^{* *}$
}

\author{
"Licenciado en Psicología. E-Mail: s_monsegur@hotmail.com \\ Elortondo 1050. (B1642CMH) San Isidro, Provincia de Buenos Aires - República Argentina. \\ "Doctor en Psicología Social. Profesor Auxiliar, Investigador del Departamento de Psicología y Coordinador del Grupo \\ de Psicología Política de la Pontificia Universidad Católica del Perú (PUCP). E-Mail: agustin.espinosa@pucp.edu.pe \\ Av. Universitaria 1801, Lima 32, Perú. \\ "'t'Licenciada en Psicología y Doctoranda en Psicología en la Universidad de Buenos Aires (UBA). Becaria Doctoral del \\ Consejo Nacional de Investigaciones Científicas y Técnicas (CONICET) y docente de Psicología Social de la Facultad de \\ Psicología de la Universidad de Buenos Aires (UBA). E-Mail: beramendimaite@psi.uba.ar \\ Thames 459, Piso 13, Dpto. B (1414) Ciudad Autónoma de Buenos Aires - República Argentina.
}

\section{RESUMEN}

El objetivo del estudio que se informa fue describir y analizar los componentes de la identidad nacional argentina y su relación con la orientación de la dominancia social (ODS) y la tolerancia a la transgresión normativa. Para tal fin, se realizaron encuestas a 170 sujetos que residían en la Ciudad Autónoma de Buenos Aires y el Conurbano Bonaerense (República Argentina). Los resultados evidencian un grado de identificación medio-alto con la categoría social argentino, a la vez que muestran una autoestima colectiva medio-alta. Los atributos autoestereotípicos que conforman la identidad nacional comprenden tres dimensiones: argentino positivo, argentino negativo y argentino avivado. Esta última dimensión fue la que presentó mayor consenso entre los participantes, lo que sugiere que la viveza es percibida como una interface entre lo negativo y lo positivo por sus características adaptativas y funcionales. Los resultados muestran además que la ODS se relaciona inversamente con los com- ponentes positivos, y directamente con los componentes negativos de la identidad nacional, lo que lleva a pensar que el ser argentino no necesariamente se vincula con el estatus o poder en personas dominantes. Finalmente, en relación con la tolerancia a la transgresión, los niveles de acuerdo con la misma son bajos en la muestra. Las caracterizaciones del argentino negativo y argentino avivado se vinculan con la tolerancia hacia ciertos comportamientos transgresores, especialmente con aquellos considerados menos graves y que no perjudican directamente a terceros.

Palabras clave: Autoestereotipos argentinos; Identidad nacional; Identidad social ambivalente; Orientación de la dominancia social; Tolerancia a la transgresión.

\section{ABSTRACT}

From early beginnings of the last century, the stereotypes and categorizations about the Argenti- 
neans where predominantly negative, both for foreigners as for Argentineans themselves (D'adamo \& García Beaudoux, 1994). This lack of a positive nationalism has been related to the lack of adherence to the norm and corruption, as well as to the bias towards the European countries shown by the Argentinean society and its members. This negative social self-concept among Argentineans is not unique in the region, in fact, a study carried out in the 90's revealed that most of Latin American countries had negative national identities (Salazar, J.M. \& Salazar, M.A., 1998). However, more recent studies carried out in countries such as Peru or Mexico suggest that there might be a shift in some aspects of their national identities, from a mainly negative one to a more ambivalent one, with some positive and some negative aspects. Some of these studies also evidenced certain relations between the national identity and other psychosocial variables such as the social dominance orientation (SDO) and the political ideology. Both high levels of SDO and a right wing political ideology had a negative impact on the different components of the national identity. These results may enlighten the study of the national identity in Argentina, that's why the aim of this study was to describe and analyze some components of the Argentinean national identity and their relationship to SDO and tolerance towards transgressive behaviors. To this end, a survey with 170 residents in Ciudad Autónoma de Buenos Aires and its surroundings was carried out. Results showed middle-high levels of identification and collective self-esteem related to the Argentinean social category. The national self-stereotypes that conform Argentinean identity were gathered in three dimensions: Positive argentine, Negative argentine, and Crafty argentine. The last dimension was the most consensual among participants, suggesting that craftiness is perceived as an interface between the negative and positive self-stereotype dimensions, because of its functionality and adaptability characteristics. The results also showed that the SDO was inversely related to the positive dimension, and directly related to the negative dimension of national identity, which suggests that the Argentine social category is not linked to status or power in dominant people. Finally, scores of tolerance toward normative transgression were low in the sample. However, negative argentine and crafty argentine dimensions were more tolerant toward transgressor behaviors that could be considered less serious and not harmful to others. The results confirm the negative relation between the SDO and the positive national identity, although the causes of this relation are yet to be studied. The research also suggests that there might be a shift in some of the components and expressions of the national identity. As well as in other countries of Latin America, this shift is characterized for its ambivalence between some positive aspects and other negative ones. Perhaps this ambivalence is best characterized by the craftiness, where the negative aspects of norm transgression and individualism meet the positive aspects of social warmth, adaptability and creativity. As for further investigations, it's important to study how the different national identities interact with specific scenarios, and how this interaction has an impact on the individual behavior. Altogether, this kind of research helps to understand the national identity more deeply, and therefore, enlighten us to make a positive change in order to develop a healthy social identity, based on mutual trust and respect.

Key words: Ambivalent social identity; Argentine self-stereotypes; National identity; Social dominance orientation; Tolerance transgression.

\section{INTRODUCCIÓN}

Una de las teorías que busca comprender la afiliación a los grupos y el mantenimiento de las identidades colectivas es la Teoría de la Identidad Social (TIS - Tajfel, 1982), la cual define a la identidad social como

"aquella parte del autoconcepto de un individuo que deriva del conocimiento de su pertenencia a un grupo (o grupos) social junto con el significado valorativo y emocional asociado a dicha pertenencia" ( $p$. 24).

Esta forma de identificación es resultado de la interacción de procesos cognitivos, evaluativos y emocionales, y constituye un proceso puente entre el comportamiento individual, la cognición social y fenómenos co- 
lectivos (Hogg \& Ridgeway, 2003). Entre las múltiples categorías sociales con las que un individuo puede sentirse vinculado, la identificación con la nación puede ser entendida como un subtipo específico de identidad social (Nigbur \& Cinnirella, 2007; Smith, Giannini, Helkama, Maczynski \& Stumpf, 2005).

Nigbur y Cinnirella (2007) encontraron que la evaluación y la caracterización del propio grupo nacional de pertenencia se da a través de la comparación intergrupal (entre naciones) y de la comparación temporal. Esta última puede operar de diferentes formas, o bien seleccionando aquellos aspectos positivos de la historia de la propia nación (ver Dias, Marques \& Páez, 1998 en Herranz \& Basabe, 1999).

Algunos atributos que caracterizan a la identidad nacional se desprenden necesariamente de la existencia del estado - nación o se ven influidos por su presencia (Pérez, 1999). En ese sentido, la identidad nacional provee a las personas: un nombre propio común que define a la comunidad, un vínculo con un territorio histórico o lugar de origen, uno o más elementos que definen una cultura compartida pública en los que se incluye la religión, las costumbres, el lenguaje, entre otros; memorias históricas compartidas que corresponden a un pasado común, una serie de derechos y obligaciones explícitos y comunes con los que deben conformarse los miembros de la nación y una economía común con cierta movilidad dentro del territorio, que es compartida por los miembros de la nación (Herranz \& Basabe, 1999).

Una de las formas de evaluar la identidad nacional es analizando los autoestereotipos de un colectivo. En esta línea, numerosas investigaciones en diversos países han tratado de identificar los contenidos autoestereotípicos relacionados con distintos grupos nacionales (D’adamo \& García Beaudoux, 1995; Marín \& Salazar, 1985; Pérez de León, 2007; Roselli, 2000; Salazar \& Marín, 1977; Salazar, J.M. \& Salazar, M.A., 1998).

Otra concepción que involucra el análisis de la identidad nacional, es la descripción y el estudio de la identidad a partir de la auto- clasificación que las personas realizan como miembros de una categoría social nacional (Salazar, J.M. \& Salazar, M.A., 1998). Esta aproximación reporta los niveles de identificación y valoración de las personas con respecto a sus grupos nacionales (c.f., Beramendi \& Zubieta, 2013; Espinosa, 2011; Espinosa \& Tapia, 2011; Laca, Mejía \& Yáñez, 2010; Pérez de León, 2007). Se ha observado que la identificación nacional construye grupos más sólidos y unidos por aparentes lazos comunes (Rottenbacher, 2008), perciben una mayor autoestima colectiva que se concreta en sentimientos de orgullo (Hewstone, Rubin \& Willis, 2002) y se asocia con autoestereotipos que aluden a la autoeficacia (Vignoles \& Moncaster, 2007; Vignoles, Regalia, Manzi, Golledge \& Scabini, 2006), o a otros atributos socialmente valorados como la sociabilidad, simpatía y calidez (Espinosa, 2011).

\section{ESTUDIOS SOBRE IDENTIDAD NACIONAL EN LATINO- AMÉRICA}

Desde la Psicología Social se aprecia un interés creciente en temas vinculados con la descripción y comprensión de la identidad nacional en los países de Latinoamérica.

Esta tradición investigativa se inicia a fines de los 70 y comienzos de los 80 (Marín \& Salazar, 1985; Salazar \& Marín, 1977). Sin embargo, en los años 90 hay una proliferación de estudios (c.f., Banchs, Cadenas, Domínguez \& Montero, 1993; D'adamo \& García Beaudoux, 1995; Herencia, 1991; Montero, 1996; Salazar, J.M. \& Salazar, M.A., 1998; Salgado, 1999) y continúa durante las siguientes décadas (c.f., Beramendi \& Zubieta, en prensa; Espinosa, 2003, 2011; Espinosa, Beramendi \& Zubieta, 2012; Espinosa \& Tapia, 2011; Laca et al., 2010; Pérez de León, 2007; Rosselli, 2000; Rottenbacher \& Espinosa, 2010). Consistentemente con la idea de que los procesos identitarios deben ser analizados dentro de un contexto social en particular, estos estudios se desarrollaron durante una transición de contenidos autoestereotípicos que sugerían una identidad 
social negativa predominante en los contextos de crisis social, política y económica que afectaron a la región en las décadas de los 80 y 90 (e.g., D’adamo \& García Beaudoux, 1995; Montero, 1996), a contenidos autoestereotípicos que hablan en la actualidad de identidades ambivalentes, mas no exclusivamente negativas (Espinosa, 2011).

En una investigación que recopila diversos resultados acerca de las identidades nacionales en América Latina, Salazar, J.M. y Salazar, M.A. (1998) encontraron que si bien los individuos se encuentran identificados con sus países y expresan un gran nacionalismo, presentan al mismo tiempo auto-imágenes nacionales fuertemente negativas. Este fenómeno se replica en la Argentina con ciertas particularidades que sugieren que la crítica hacia lo nacional y lo latinoamericano es más fuerte que en otros países de la región (D' adamo \& García Beaudoux, 1995; Roselli, 2000).

Al respecto, D'adamo y García Beaudox (1995) plantearon la existencia de un nacionalismo crítico que se asemeja a la estrategia de superación de la identidad negativa denominada movilidad social (Tajfel, 1982; Tajfel \& Turner, 1979). El nacionalismo crítico se refiere a una actitud que combina una fuerte crítica hacia el propio país y la identificación con éste. De esta forma, al criticar aquellos aspectos que considera negativos, la persona queda liberada de ellos y se presenta como distinta del argentino prototípico.

Consistentemente con lo anterior, la estrategia de movilidad social en América Latina se pone en evidencia cuando las personas tienden a enfatizar su origen foráneo y a definirse a sí mismas como europeas o identificarse intensamente con éstos (Espinosa, 2011; Roselli, 2000). Lo anterior supone un distanciamiento psicológico y la atenuación de la pertenencia a países latinoamericanos como grupos de bajo estatus, en un proceso que desde la Psicología Social latinoamericana se denominó altercentrismo (Montero, 1994). Este fenómeno no deja exenta a la Argentina (D'adamo \& García Beaudoux, 1995; Roselli, 2000; Salazar, J.M. \& Salazar, M.A., 1998) debido principalmente a la corriente inmi- gratoria europea que tuvo hasta mediados del Siglo XX. Se ha sugerido que esta tendencia del argentino a identificarse con el europeo genera una especie de complejo de inferioridad, pero al mismo tiempo le permite ubicarse por encima del resto de Latinoamérica (Salazar, J.M. \& Salazar, M.A., 1998).

Analizando los contenidos autoestereotípicos específicos sobre los argentinos se encuentra una evolución similar a la observada en el resto de América Latina, que va desde una marcada negatividad en la década de los 90 , con atributos marcadamente negativos como corruptos y poco trabajadores (D'adamo \& García Beaudoux, 1995; Salazar, J.M. \& Salazar, M.A., 1998), a una constitución de la identidad más ambivalente en las siguientes décadas (Beramendi \& Zubieta, 2013; Roselli, 2000).

La ambivalencia identificada en los estudios citados se expresa, por un lado, en una visión positiva de los argentinos, vistos como cálidos, solidarios, inteligentes, flexibles, reflexivos, amistosos y abiertos (Beramendi \& Zubieta, 2013; Roselli, 2000) mientras que por otra parte, se los vincula a importantes defectos que están a su vez muy relacionados entre sí: prepotentes, egoístas, omnipotentes, vivos, piolas, cuestionadores, vagos y corruptos (Beramendi \& Zubieta, 2013; D'adamo \& García Beaudoux, 1995; Roselli, 2000). Según Roselli (2000), es razonable que esta autopercepción ambivalente genere una elevada autoestima, a la vez que mantiene una crítica desvalorizante del sí mismo colectivo nacional.

De los atributos mencionados previamente, el de la viveza merece un apartado especial. La persona viva o piola es quien transgrede una norma, no es sancionado por dicha acción y es valorado positivamente, a tal punto que quien no infringe la norma es percibido como tonto (Espinosa, 2003). Así la transgresión se convierte en una característica idiosincrática que se asume como necesaria para operar en el contexto local (Beramendi \& Zubieta, 2013). Esta característica es consistente con estudios que dan cuenta que en la Argentina existe una creencia generalizada que reconoce que el incumplimiento de la 
ley es poco o nada grave, o que la mayoría de los ciudadanos no cumple con la ley (Beramendi \& Zubieta, en prensa; Latinobarómetro, 2006, 2008, 2009, 2010).

En consonancia con estas creencias, se ha corroborado que las personas en el contexto argentino perciben niveles altos de transgresión que se plasman en todo el entramado de la sociedad, por lo cual no se da la vivencia como un hecho aislado sino como una pauta de comportamiento social compartido, pero considerado ilegal. La transgresión se asocia, entre diversas variables, a la percepción de falta de legitimidad de las instituciones y de las autoridades y a la debilidad normativa (Alonso \& Brussino, 2012; Beramendi \& Zubieta, 2013). Sautú, Bonolio y Perugorría (2004) observaron que los argentinos son más tolerantes ante ciertos tipos de transgresiones. Hay una mayor indulgencia ante la micro-corrupción en comparación con la macro-corupción, ya que la consideran menos grave, inevitable y perteneciente a sus prácticas cotidianas. Sienten la necesidad de involucrarse en pequeñas irregularidades, o bien eligen la opción de usar estratégicamente circuitos corruptos para obtener ventajas y ahorrarse mayores problemas.

Lo anterior sugiere que al estudiar las características específicas de los miembros de un grupo, es necesario vincularlas a las representaciones sociales que se tengan del país y su situación socio-política. En este sentido, se encuentra una relación entre la visión negativa sobre el sistema normativo argentino y las representaciones sobre la identidad nacional, observándose una estrecha relación entre las descripciones que se realizan del sistema y las características de los ciudadanos que en él participan (Beramendi \& Zubieta, 2013). La percepción de ineficacia e injusticia en el sistema genera una sensación de desamparo en la ciudadanía y estimula estrategias y prácticas individualistas de resolución de problemas cotidianos, que muchas veces comprenden la transgresión de las normas impuestas. En ese sentido, los ciudadanos incorporan en su auto-representación, características como las de sentirse omnipotentes, ser cuestionadores de las normas y egoístas, en- tre otras. Asimismo, muchos de los adjetivos que surgen como positivos tales como ser flexible, adaptativo o reflexivo, también responden a este contexto de incertidumbre e inoperancia (Beramendi \& Zubieta, en prensa).

\section{ORIENTACIÓN DE LA DOMINANCIA SOCIAL E IDENTI- DAD SOCIAL}

La Teoría de la Dominancia Social (TDSPratto, Sidanius, Stallworth \& Mallé, 1994; Sidanius \& Pratto, 1999) busca explicar y entender el proceso de producción y mantenimiento del prejuicio y la discriminación teniendo en cuenta múltiples niveles de análisis. No sólo considera las variables individuales, sino que también incluye un análisis en el cual intervienen las ideologías, las políticas, las prácticas institucionales y las relaciones que se dan entre los individuos (Delfino \& Zubieta, 2011; Pratto, Sidanius \& Levin, 2006; Pratto et al., 1994). Los autores parten de la premisa de que, sin importar la forma de gobierno, los contenidos o el sistema de creencias, las sociedades humanas que generan un superávit económico tenderán a organizarse sobre jerarquías basadas en grupos en las que al menos uno de ellos gozará de un mayor estatus social y poder que los demás. Para reducir el conflicto que genera esta desigualdad y así garantizar su supervivencia, las sociedades crean mitos de legitimación que sostienen la desigualdad resaltando la superioridad de un grupo por sobre otro. Es en estos mitos donde se encuentra la raíz del prejuicio étnico, el sexismo y el conservadurismo político, entre otros (Pratto et al., 2006). La aceptación de los mitos que legitiman y refuerzan las jerarquías y la desigualdad está, en parte, determinada por el deseo general de los individuos de mantener relaciones intergrupales jerárquicas y no igualitarias. Es esta motivación la que los autores de la TDS buscan evaluar con la Escala de Orientación a la Dominancia Social (ODS Pratto et al., 1994).

Articulando ambas teorías (Teoría de la Identidad Social y Teoría de la Dominancia 
Social) se ha encontrado que, si bien se espera que el deseo por mantener una identidad social positiva manifieste un afecto positivo hacia el endogrupo, los deseos de dominación social se manifestarán en un afecto positivo hacia el grupo dominante, y en un menor afecto hacia el grupo de menor estatus, sin importar el grupo al que uno pertenezca (Espinosa, Calderón, Burga \& Güimac, 2007; Levin \& Sidanius, 1999; Schmitt, Branscombe \& Kappen, 2003). En esta misma línea se ha argumentado que la construcción saludable de una identidad colectiva nacional resulta difícil en un contexto donde el prejuicio y la exclusión de importantes segmentos de la sociedad predominan, debido a que la desvalorización de las minorías cuestiona la imagen global de la nación (Espinosa, 2011). Es así que dependiendo de la sociedad y la cultura, los procesos involucrados en la construcción de la identidad social pueden, o bien generar una identidad nacional saludable y unificada o una frágil y segmentada.

Con respecto a las relaciones entre la ODS y la construcción de la identidad nacional en el contexto latinoamericano, en Perú se ha observado que la ODS se asocia inversamente a la valoración del grupo nacional como así también a la valoración de los grupos minoritarios o de menor estatus. Espinosa (2011) ha argumentado que para estos individuos, el grupo nacional es percibido como de bajo valor instrumental y estatus, por ende perjudica sus aspiraciones de dominación y logro, y es por ello que están motivados a distanciarse del mismo. Asimismo, las personas con niveles mayores de ODS serían más tolerantes a ciertos comportamientos transgresores, en especial hacia aquellos relacionados con el uso de conocidos, ya que al utilizar los contactos personales en favor propio o de otros miembros del endogrupo, éstos estarían cumpliendo su meta de dominación (Rottenbacher \& Schmitz, 2012).

\section{OBjetIVOS}

A partir de las concepciones negativas de la identidad argentina que se asocian a la ba- ja moralidad y a la transgresión se consideró relevante estudiar su relación con la tolerancia a la transgresión y la orientación a la dominancia social. Basándose en los hallazgos hasta aquí descritos, el trabajo de investigación realizado indagó acerca de las diferentes manifestaciones de la identidad nacional en la Argentina tales como el grado de identificación con los argentinos, los autoestereotipos asociados a éstos y el sentimiento de orgullo de pertenecer a dicho colectivo. Adicionalmente se relacionaron las medidas de identidad nacional con las dos variables psicosociales mencionadas previamente, la ODS (Pratto, et al., 1994; Sidanius \& Pratto, 1999) y la tolerancia a la transgresión (Rottenbacher \& Schmitz, 2012).

\section{MÉTOdo}

El estudio que se informa es no experimental y transversal, también es descriptivo, correlacional y de diferencias de grupos.

\section{Participantes}

La muestra es intencional y estuvo compuesta por 170 participantes argentinos (43.5\% mujeres), residentes en la Ciudad Autónoma de Buenos Aires y en el Conurbano Bonaerense, cuyas edades fluctúan entre los 18 y 65 años $(M=27.75, D E=9.95)$. El $4.1 \%$ pertenece a la clase media-baja, el $42.9 \%$ a la clase mediamedia, el $51.8 \%$ media-alta y el $1.2 \%$, alta.

\section{INSTRUMENTOS}

\section{1.- Escala de Autoestima Colectiva Argentina}

Se adaptaron los ítemes de la Subescala de Autoestima Colectiva Privada de Luhtanen y Crocker (1992) a la categoría social argentino. La subescala consta de 4 ítemes que evalúan la relación afectiva de los participantes con su identificación con la categoría Argentino a partir de ítemes tales como: 'En general, me siento afortunado por ser argentino' o 'Generalmente siento que ser argenti- 
no no merece la pena'. Los valores de respuesta de esta escala son de 1 a 5 (1: totalmente en desacuerdo y 5: totalmente de acuerdo). El coeficiente de consistencia interna de la escala es igual a .77.

\section{2.- NIVEL DE IDENTIFICACIÓN CON LA CATEGORÍA ARGENTINO}

Esta medida se obtiene a través de un ítem que plantea la siguiente pregunta: ‘¿Cuál es su grado de identificación con los argentinos?'. Los participantes respondieron en una escala de 1 a 5 (1: nada y 5: mucho).

\section{3.- Escala de Autoestereotipo Colectivo AR- GENTINO}

Se adaptó al contexto argentino la escala desarrollada por Espinosa (2003) en el contexto peruano tomando como referencia diversos estudios acerca de la identidad nacional desarrollados en este país (Beramendi \& Zubieta, 2013; D’adamo \& García Beaudoux, 1995; Roselli, 2000). Luego se elaboró una lista de 35 adjetivos que responden a cómo se perciben los argentinos. Cada adjetivo fue valorado por los participantes en una escala del 1 al 5 (1: totalmente en desacuerdo y 5: totalmente de acuerdo). En el apartado Resultados se presentan las dimensiones que arrojó el análisis factorial exploratorio y la confiabilidad interna de cada una de ellas. Para poder llevar a cabo el análisis factorial exploratorio, se aplicaron alrededor de cinco cuestionarios por cada adjetivo de la escala.

\section{4.- Escala de ORIENTACIÓN a La Dominancia So- CIAL (PRATTO ET AL., 1994)}

Esta escala cuenta con 16 enunciados del tipo: 'El valor que tienen algunos grupos de personas es mayor que el de otros', 'Deberíamos hacer todo lo posible para igualar las condiciones de los diferentes grupos', 'A veces es necesario utilizar la fuerza contra otros grupos para conseguir lo que tu grupo quiere'.
Las opciones de respuesta van de 1 a 7 (1: totalmente en desacuerdo y 7: totalmente de acuerdo). Los valores que superan el punto medio del valor de la escala muestran una mayor tendencia a la orientación de la dominancia social. El coeficiente de consistencia interna de la escala es Alpha igual a .79.

\section{5.- Escala de TOLERANCIA A LA TRANSGRESIÓN}

La versión original de este instrumento está conformada por 24 ítemes que presentan diversos comportamientos transgresores e ilegales (Rottenbacher \& Schmitz, 2012). El participante debe indicar qué tan tolerable o intolerable le resulta cada uno de los comportamientos presentados en una escala tipo Likert de 7 puntos (1: completamente inaceptable y 7: totalmente aceptable tolerable).

Se utilizaron las dimensiones propuestas por los autores para agrupar los diversos comportamientos transgresores en dimensiones específicas: Uso de dinero / Sobornos $(\alpha=.46)$, Transgresión a las leyes $(\alpha=.52)$, Uso de contactos e influencias $(\alpha=.75)$ y Transgresión a las convenciones sociales $(\alpha=.62)$. Cabe resaltar que en su estudio, los autores eliminaron de la conformación original de la escala los ítemes 1, 5 y 23. En el estudio que se informa esos ítemes fueron descartados del análisis junto a los ítemes 4 y 16 por presentar bajas cargas factoriales. En conjunto, los ítemes eliminados afectaban los niveles de confiabilidad de todas las dimensiones de la escala y en algunos casos no correspondían contextualmente a situaciones identificables por los participantes del estudio. A pesar de su baja confiabilidad en el contexto local se decidió aplicar esta escala ya que no se encontraron otras que indagaran esta temática.

\section{6- DATOS SOCIODEMOgRÁFICOS}

La ficha de registro de datos sociodemográficos contenía preguntas relativas a la edad, sexo y la autoclasificación según clase socioeconómica. 


\section{Procedimiento}

Los cuestionarios fueron auto-administrados y su aplicación se realizó a 40 sujetos mediante encuestas presenciales en formato papel y a 130 sujetos que respondieron las encuestas electrónicamente en el formato google.docs. Se complementó el estudio con esta técnica porque, a pesar que ha sido incorporada hace poco tiempo, se la reconoce como un método igual o mejor que la recolección de datos auto-administrados y presenciales (Lyons, Cude, Lawrence \& Gutter, 2005). Este procedimiento aumenta la rapidez de recolección de datos, la diversidad de la muestra, reduce los costos (Weber \& Bradley, 2006), disminuye la deseabilidad social y los errores procedimentales correspondientes a la carga de datos (Lyons et al., 2005), entre otras ventajas. En ambos casos, los cuestionarios fueron acompañados de una hoja de consentimiento informado que daba cuenta del propósito general del estudio, el carácter voluntario de la participación en el mismo, el respeto por la confidencialidad de las respuestas, la salvaguarda del anonimato de los participantes y el uso de la información con fines estrictamente académicos.

Una vez recopiladas las respuestas, éstas fueron procesadas a través del paquete estadístico SPSS 18.

\section{ANÁLISIS DE LOS DATOS}

Los resultados de los análisis de normalidad indicaron que no había una distribución normal en todas las escalas. Se controló la severidad de la asimetría y como se hallaron dentro de los niveles leves, se utilizaron pruebas paramétricas por la robustez de los análisis y las preguntas de investigación (Stanley \& Glass, 1986).

Para realizar los análisis correspondientes a la Escala de Autoestereotipo Colectivo Argentino se utilizaron las siguientes técnicas: (a) análisis factorial exploratorio (AFE, método de extracción de ejes principales y rotación Varimax) para obtener las dimensiones de la escala y su validez interna, (b) Alpha de Cronbach para analizar la fiabilidad de los factores de la escala, (c) análisis de conglomerado de K-Medias que permitió segmentar las dimensiones obtenidas con el análisis factorial en dos grupos, principalmente para indagar si la dimensión Argentino avivado (dimensión más ambigua) se asociaba a una visión positiva o negativa y (d) correlaciones $r$ de Pearson entre las variables de identidad, ODS y Tolerancia a la Transgresión.

Para analizar la Escala de Tolerancia a la Transgresión, se utilizó el análisis ANOVA de medidas repetidas a fin de identificar si había diferencias significativas entre sus cuatro dimensiones.

\section{Resultados \\ IDENTIDAD NACIONAL ARGENTINA}

Los resultados muestran que los participantes reportan una autoestima colectiva medio-alta $(M=3.89, D E=.88)$, así como también un nivel de identificación con los argentinos medio-alto $(M=3.83, D E=.97)$.

En relación con los contenidos autoestereotípicos, los 35 atributos presentados a los participantes fueron sometidos a un análisis factorial exploratorio que arrojó tres dimensiones $(K M O=.798$; Test de Esfericidad de Bartlett: $\chi^{2}=2004.445, g l=595, p<.001$ ) que explicaban el $38.2 \%$ de la variancia total (ver Tabla 1). El primer factor se denominó Argentino positivo y tuvo un índice de consistencia interna alto $(\alpha=.85)$, el segundo se denominó Argentino negativo y mostró una consistencia interna aceptable $(\alpha=.79)$ y el tercer factor se denominó Argentino avivado $\mathrm{y}$ presentó un nivel de consistencia interna aceptable $(\alpha=.70)$.

A nivel descriptivo, los niveles promedio de acuerdo con las dimensiones del autoestereotipo argentino muestran que dos de estas dimensiones se ubicaron por encima del punto medio de la escala y fueron las siguientes: Argentino positivo $(M=3.13$, $D E=.56)$ y Argentino avivado $(M=4.08$, 
$D E=.54)$. Por otra parte la dimensión Argentino negativo $(M=2.99, D E=.57)$ evidencia un nivel de acuerdo menor entre los participantes.

A partir de las tres dimensiones factoriales descritas, se realizó un análisis de Conglomerado de K-Medias que permitió segmentar a los participantes en dos grupos: Optimistas y Pesimistas. Los niveles de acuerdo con las dimensiones autoestereotípicas por grupo se reportan en la Tabla 2.

La Tabla 2 sugiere que los Optimistas reportaron un acuerdo alto con la noción del Argentino avivado, un acuerdo medio-alto con la noción del Argentino positivo y un acuerdo medio con la noción del Argentino negativo. En contraposición, los Pesimistas reportaron un acuerdo medio-alto con la idea del Argentino avivado, un acuerdo medio alto con la noción del Argentino negativo y un acuerdo medio con la dimensión del Argentino positivo.

Posteriores análisis de correlación sugieren relaciones significativas entre los componentes de la identidad nacional argentina. En ese sentido, la autoestima colectiva se relacionó de manera positiva con el grado de identificación $[r(168)=.466, p<.01]$. Asimismo, la autoestima colectiva se relacionó directamente con las dimensiones estereotípicas del Argentino positivo $[r(168)=.397, p<.01]$, y del Argentino avivado $[r(168)=.159, p<.05]$, e inversamente con la dimensión del Argentino negativo $[r(168)=-.421, p<.01]$. El grado de identificación con la Argentina se relacionó directamente con las dimensiones estereotípicas del Argentino positivo $[r(168)=.433, p<.01]$ $\mathrm{y}$ del Argentino avivado $[r(168)=.217$, $p<.01]$ e inversamente con la dimensión del Argentino negativo $[r(168)=-.312, p<$ $.01]$.

Los contenidos autoestereotípicos también arrojaron relaciones entre sí. De manera específica, la dimensión del Argentino positivo se relacionó directamente con la dimensión del Argentino avivado $[r(168)=.332$, $p<.01]$, y de manera indirecta con la dimensión del Argentino negativo $[r(168)=-.263$, $p<.01]$. No se reportaron relaciones significativas entre las dimensiones de Argentino negativo y Argentino avivado.
ORIENTACIÓN DE LA DOMINANCIA SOCIAL Y TOLERANCIA A LA TRANSGRESIÓN

Los resultados muestran que los participantes de este estudio exhibieron un nivel bajo de orientación a la dominancia social, por debajo del punto medio de la escala de medición $(M=2.72, D E=.92)$.

A nivel descriptivo los niveles promedio de acuerdo con respecto a la transgresión de los diferentes tipos de norma se ubicaron en todos los casos por debajo del punto medio de la escala y fueron los siguientes: Tolerancia al uso de dinero en beneficio propio $(M=1.44$, $D E=.53)$, Tolerancia a la transgresión de leyes $(M=1.91, D E=.95)$, Tolerancia al uso de conocidos en beneficio propio $(M=2.64$, $D E=1.23)$ y Tolerancia a la transgresión de convenciones sociales $(M=3.14, D E=1.11)$.

Se calculó un ANOVA de medidas repetidas para identificar si había diferencias significativas entre las cuatro dimensiones de tolerancia a la transgresión. Los resultados llevaron a rechazar la hipótesis de esfericidad, por lo cual se aplicó la corrección de grados de libertad propuesta por Greenhouse-Geisser. Los resultados mostraron que existen diferencias entre las puntuaciones de las dimensiones evaluadas $\left[F(2.735,169)=164.144, \eta^{2}=.49\right.$, $p<.001]$. Específicamente, para examinar el contraste entre pares de categorías se aplicó la Prueba de Bonferroni, la cual mostró que existían diferencias significativas entre todos los pares evaluados $(p<.001)$.

RELACIONES ENTRE IDENTIDAD NACIONAL, ORIENTACIÓN A LA DOMINANCIA SOCIAL Y TOLERANCIA A LA TRANSGRESIÓN

En primer lugar, se encontró una asociación inversa entre la dimensión de Argentino positivo y la Dominancia social $[r(164)=-.241, p<.01]$. En cambio, la dimensión Argentino negativo se relacionó de forma directa con la ODS $[r(164)=.289$, $p<.001]$. No se apreciaron relaciones específicas entre el grado de identificación con la Argentina y la dimensión del Argentino avi- 
vado con ODS, aunque sí se observó una relación inversa entre la Autoestima colectiva y la escala de ODS $[r(166)=-.244$, $p<.01]$.

Los componentes de la identidad nacional reportaron pocas relaciones con las dimensiones de la Tolerancia a la transgresión. De manera específica, la imagen del Argentino negativo se relacionó directamente con el uso de dinero $[r(166)=.183, p<.05]$ y la Tolerancia al uso de conocidos para el beneficio personal $[r(166)=.162, p<.05]$. Por otra parte, la imagen del Argentino como avivado se relacionó con la Tolerancia al uso de conocidos para el beneficio personal $[r(168)=.210$, $p<.01]$. No se observaron correlaciones significativas entre el grado de identificación, la autoestima colectiva y la imagen del Argentino positivo con algún tipo de tolerancia a la transgresión.

La escala ODS se asoció directamente con dos dimensiones de la Tolerancia a la transgresión que fueron: tolerancia al uso de dinero en beneficio propio $[r(166)=.247, p<.01]$ y Tolerancia al uso de conocidos $[r(166)=.261$, $p<.01]$.

Comparaciones de MEdiAs SEgÚN PERFILES DE ARGENTINOS: LOS OPTIMISTAS VERSUS LOS PESIMISTAS

Se analizaron los perfiles de argentinos Optimistas y Pesimistas en función de la autoestima colectiva, el grado de identificación, la ODS y la tolerancia hacia la transgresión. En cuanto a la autoestima colectiva, se encontraron diferencias significativas a favor de los Optimistas $[t(140.067)=4.881$, $p<.001, M_{\text {optimistas }}=4.19, D E=.76$ versus $\left.M_{\text {pesimistas }}=3.54, D E=.91\right]$, lo mismo que ocurre al comparar el grado de identificación con la Argentina entre ambos grupos $\left[t(163)=5.964, p<.001, M_{\text {optimistas }}=3.74\right.$, $D E=.88$ versus $M_{\text {pesimistas }}=2.90$, $D E=.92]$. En cuanto a la ODS, ésta resulta inferior en los Optimistas con respecto a los
Pesimistas $[t(161)=-2.301, p<.05$, $M_{\text {optimistas }}=2.57, D E=.87$ versus $\left.M_{\text {pesimistas }}=2.91, D E=.97\right]$. Los grupos de Optimistas y Pesimistas no se diferencian significativamente en sus niveles de tolerancia hacia los distintos tipos de transgresión evaluados.

\section{Discusıón}

IDENTIDAD NACIONAL ARGENTINA: DE LA NEGATIVIDAD A LA AMBIVALENCIA

Durante la década de los años 90, las identidades nacionales descritas en diferentes estudios en Latinoamérica demostraron poseer contenidos predominantemente negativos (cf., Banchs et al., 1993; D’adamo \& García Beaudoux, 1995; Herencia, 1991; Montero, 1996; Salazar, J.N. \& Salazar, M.A., 1998; Salgado, 1999), lo cual parece estar vinculado a los contextos de crisis socio-política y económica que aquejaban a la región en esa época. Estudios posteriores muestran una evolución del sesgo abiertamente negativo sobre las identidades colectivas en los países de la región hacia representaciones más ambivalentes (cf., Espinosa, 2011; Laca et al., 2010). Estudios en la Argentina replican la tendencia de una evolución que va de la negatividad hacia la ambivalencia.

En los primeros estudios en el contexto argentino, D'adamo y García Beaudoux (1995) encontraron una descripción del argentino como una persona corrupta, inestable y subdesarrollada. Posteriormente, Roselli (2000) encontró que si bien los atributos negativos relativos a la corrupción y a la poca laboriosidad seguían apareciendo, otros atributos ligados a la inteligencia y a la sociabilidad aparecían dentro de los más utilizados para describir al argentino. Más recientemente, Beramendi y Zubieta (2013) observaron que persisten elementos negativos, aunque comienzan a aparecer contenidos positivos relacionados con la solidaridad, la capacidad adaptativa y la sociabilidad. Estos resultados sugieren que en este proceso de cambio de los 
contenidos autoestereotípicos, no sólo han aparecido atributos positivos, también en algunos atributos negativos se ha ido atenuando la connotación peyorativa que originalmente poseían y han pasado a tener una connotación ambivalente. Esta ambivalencia se aprecia en el caso de la viveza, en ella sus efectos no deseados siguen vigentes, pero a la vez es percibida como adaptativa y necesaria para salir adelante en el contexto argentino.

Consistentemente con lo anterior, los resultados del estudio realizado muestran que la categorización predominante del argentino es la de alguien con una buena capacidad adaptativa, creatividad, sociabilidad y capacidad de expresarse, atributos que podrían ser aplicados para sacar alguna ventaja o provecho personal, por lo cual se lo caracteriza también como una persona viva y chamuyera (charlatana).

Además de esta imagen del argentino avivado, también surgieron otras dos categorizaciones, una netamente positiva y la otra negativa. Dentro de la visión positiva se describe al argentino como una persona de alto valor instrumental, exitoso e inteligente, con un elevado sentido de la moral y respeto por el prójimo, alegre y comprometido tanto con los demás miembros de la sociedad como con su patria. Por el contrario, la visión pesimista del argentino está compuesta por adjetivos que lo describen como un individuo de bajo valor instrumental debido tanto a una falta de compromiso y tendencia al ocio como a una falta de capacidad. Adicionalmente, se lo describe también como corrupto, indigno de confianza, melancólico e individualista al punto tal de no comprometerse con la defensa de su patria.

En general, la identificación con el argentino y el nivel de autoestima colectiva son elevados, existiendo una relación directa entre ambas variables, lo que corrobora la hipótesis de la autoestima descrita por Abrams y Hogg (1988), según la cual, el nivel de autoestima que despierta la pertenencia a un grupo será mayor cuanto mayor sea el nivel de identificación con el mismo.

Continuando con el análisis de los componentes de lo que constituye la identidad nacional, se observaron algunas relaciones entre los niveles de identificación y autoestima y la autocategorización que permiten sugerir la presencia de una consistencia cognitivo-afectiva entre la auto-representación, la identificación y los juicios de valor que se hagan sobre el endogrupo nacional. En este sentido, en la medida en que se categorice al argentino positivamente, la autoestima colectiva y el grado de identificación con el argentino serán mayores; en cambio, la categorización negativa mitigará la autoestima colectiva y el grado de identificación con los argentinos.

Segmentos de ARgENTINOS: Los Optimistas VerSUS LOS PESIMISTAS Y LA CONSISTENCIA DEL ATRIBUTO AVIVADO

A partir de los niveles de acuerdo con respecto a las diferentes dimensiones del autoestereotipo colectivo del argentino (positivo, negativo y avivado), se pudo agrupar a los participantes en dos categorías, los Optimistas y los Pesimistas. El grupo de los Optimistas obtuvo puntuaciones más elevadas en las dimensiones de Argentino positivo y del Argentino avivado. Por su parte, el grupo de los Pesimistas obtuvo puntuaciones más elevadas en la dimensión del Argentino negativo y también presentó puntuaciones elevadas en la dimensión del Argentino avivado, aunque con menor intensidad que las puntuaciones obtenidas por los optimistas. Esto sugiere que existe un alto grado de consenso acerca de la categorización del argentino como una persona viva, tanto en quienes predomina una visión pesimista como en aquellos en quienes predomina una visión optimista de lo que constituye ser argentino. Como se mencionó anteriormente, esto demuestra el giro en lo que constituye la viveza, de una visión marcadamente negativa a una más ambivalente. Lo que se podría explicar aludiendo a que los atributos que conforman esta dimensión operan en el límite de lo funcional y lo moral. Lo anterior es coherente con la relación entre esta dimensión autoestereotípica y 
la tolerancia al uso de amigos y conocidos en beneficio personal, pues esta expresión de la transgresión es percibida como necesaria pero menos dañina ante terceros (Beramendi \& Zubieta, 2013).

Otro aspecto que merece la pena remarcar con respecto a la agrupación entre optimistas y pesimistas es que si bien los pesimistas presentan niveles de autoestima colectiva y de identificación más bajos que los optimistas, las puntuaciones obtenidas por este grupo son medias-altas.

ORIENTACIÓN DE LA DOMINACIÓN SOCIAL: EXPRESIÓN NEGATIVA DE LA IDENTIDAD NACIONAL ARGENTINA

En cuanto a la relación entre la ODS y la identidad nacional, en países latinoamericanos se ha encontrado que uno de los aspectos que menoscaba la constitución de las identidades nacionales tiene que ver con la gestión de la diversidad étnica y cultural en contextos de inequidad y exclusión social (Espinosa, 2011). Sin embargo, el marcado origen europeo y la relativa homogeneidad cultural (c.f., Romero \& Bertoni, 1989), social y económica de la sociedad argentina en comparación con otras sociedades latinoamericanas, y en particular la homogeneidad de la muestra estudiada, sugieren que la relación negativa entre ODS y los componentes positivos de la identidad no se explican como un problema de gestión de la diversidad. Estas relaciones podrían estar vinculadas al problema del altercentrismo en los términos descritos por Montero (1994) pues se aprecia en los argentinos una mirada constante y una valoración positiva de lo europeo en desmedro de los aspectos que vinculan a la nación con la región latinoamericana. Esto posicionaría a la Argentina en un punto intermedio entre el ser una nación europea, como un aspecto desiderativo no siempre alcanzado y una nación latinoamericana, de la cual los argentinos no siempre se sienten parte (Roselli, 2000; Salazar, J.M. \& Salazar, M.A., 1998).
TOLERANCIA A LA TRANSGRESIÓN NORMATIVA: DESEABILIDAD SOCIAL

En base a estudios previos que indicaban una percepción generalizada de conductas transgresoras y un sistema normativo laxo, deslegitimado y poco eficiente (Beramendi \& Zubieta, 2013, en prensa; Latinobarómetro, 2006, 2008, 2009, 2010), en la investigación realizada se esperaba encontrar niveles altos de tolerancia a la transgresión. Sin embargo, los datos obtenidos no respaldan esta expectativa, evidenciándose puntuaciones más bien bajas en la tolerancia a conductas transgresoras. A pesar de ello, las correlaciones entre la Escala de Autoestima Colectiva Argentina y la Escala de Tolerancia a la Transgresión sugieren que los estereotipos del Argentino negativo se asocian al uso del dinero y de conocidos para el beneficio personal, y los del Argentino avivado se relacionaron con la tolerancia al uso de conocidos para el beneficio personal. Esto da cuenta de cómo el uso de los conocidos termina siendo una estrategia asociada al estereotipo negativo del argentino, pero al mismo tiempo, se lo vincula con el Argentino avivado, por lo cual le confiere un carácter funcional y adaptativo. Como planteaban Sautú y colaboradores (2004) hay ciertos tipos de comportamientos que por considerarlos necesarios son tolerados y así disminuye su connotación de baja moralidad.

En conclusión, la relación entre la ODS y la tolerancia a la transgresión mostró que la primera se relaciona sólo con unas pocas expresiones de tolerancia a la transgresión. Esto respalda parcialmente la idea de que la dominancia social representa un factor de riesgo en la práctica de comportamientos transgresores, pues los que se perciben como más poderosos o dominantes eventualmente creen que pueden actuar con mayor impunidad (Rottenbacher \& Schmitz, 2012).

\section{LIMITACIONES, REFLEXIONES FINALES Y FUTURAS INVESTIGACIONES}

Con respecto a las limitaciones del estudio realizado se debe mencionar por un lado la se- 
lección y el tamaño de la muestra, la cual presenta una elevada homogeneidad en lo que se refiere al lugar de residencia (sectores de nivel socioeconómico medio y medio alto de la Ciudad Autónoma de Buenos Aires y Conurbano Bonaerense). En futuras investigaciones sería interesante evaluar la manifestación de la identidad nacional considerando poblaciones de diferentes niveles socioeconómicos y de todas las regiones de Argentina, especialmente teniendo en cuenta la clásica división antes mencionada entre aquellos individuos que habitan en la Ciudad Autónoma de Buenos Aires y Conurbano Bonaerense y quienes lo hacen en el interior del país.

Otra limitación fue impuesta por la Escala de Tolerancia a la Transgresión, que demostró tener bajos niveles de confiabilidad. Con la finalidad de poder continuar estudiando la relación de los individuos con la norma, se plantea como desafío para futuras investigaciones, el desarrollo de instrumentos que atenúen la deseabilidad social en la evaluación de este tema, ya que en general en las escalas que evalúan aspectos donde se establece una auto-presentación, existe cierta estabilidad en las respuestas, en este caso de rechazo a la transgresión, lo que estaría afectando a los indicadores de consistencia interna de la escala utilizada (Mezulis, Abramson, Hyde \& Hankin, 2004).

Una posible línea de investigación futura podría ser la exploración de la categoría Argentino avivado. Sería de interés ahondar en el origen y contenidos de este autoestereotipo, tanto en aquellos individuos que lo perciben negativa como positivamente, a fin de poder elaborar estrategias que ayuden a resignificar la idea de viveza en una idea de creatividad que gire en torno a atributos positivos y funcionales que permitan resolver aquellas expresiones comportamentales que hacen de la viveza algo inmoral. Esto último es importante porque el desarrollo de una identidad nacional saludable genera relaciones de confianza y respeto que incrementan la cohesión y permite el desarrollo del capital social. 
TABLA 1

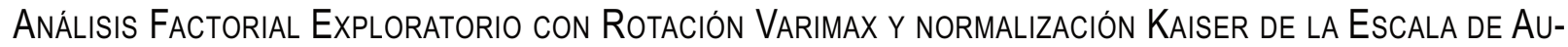
TOESTEREOTIPO ARGENTINO

\begin{tabular}{|c|c|c|c|}
\hline Itemes & Factor 1 & Factor 2 & Factor 3 \\
\hline Los argentinos somos: De confianza / Confiables & .717 & & \\
\hline Los argentinos somos: Cumplidos / Cumplidores & .671 & & \\
\hline Los argentinos somos: Exitosos & .656 & & \\
\hline Los argentinos somos: Honrados & .621 & & \\
\hline Los argentinos somos: Considerados / Respetuosos & .607 & & \\
\hline Los argentinos somos: Honestos & .607 & & \\
\hline Los argentinos somos: Desarrollados & .596 & & \\
\hline Los argentinos somos: Emprendedores & .578 & & \\
\hline Los argentinos somos: Trabajadores & .524 & & \\
\hline Los argentinos somos: Solidarios & .489 & & \\
\hline Los argentinos somos: Patriotas & .489 & & \\
\hline Los argentinos somos: Capaces / Inteligentes & .481 & & \\
\hline Los argentinos somos: Alegres & .473 & & \\
\hline Los argentinos somos: Cuestionadores / No conformistas & .276 & & \\
\hline Los argentinos somos: Incumplidos / No cumplidores & & .660 & \\
\hline Los argentinos somos: Prepotentes / Abusivos & & .628 & \\
\hline Los argentinos somos: Individualistas / Egoístas & & .556 & \\
\hline Los argentinos somos: Ociosos / Vagos & & .549 & \\
\hline Los argentinos somos: Corruptos & & .536 & \\
\hline Los argentinos somos: Mentirosos & & .530 & \\
\hline Los argentinos somos: Subdesarrollados & & .525 & \\
\hline Los argentinos somos: Conformistas / Resignados & & .492 & \\
\hline Los argentinos somos: No quieren a su patria & & .484 & \\
\hline Los argentinos somos: Incapaces / Tontos & & .481 & \\
\hline Los argentinos somos: Fracasados & & .480 & \\
\hline Los argentinos somos: Tristes / Melancólicos & & .421 & \\
\hline Los argentinos somos: Poco confiables & & .323 & \\
\hline Los argentinos somos: Sociables & & & .719 \\
\hline Los argentinos somos: Expresivos / Comunicativos & & & .596 \\
\hline Los argentinos somos: Creativos & & & .549 \\
\hline
\end{tabular}

(Contínúa) 
Identidad nacional, dominancia social y tolerancia a la transgresión

TABLA 1 (CONTINUACIÓN)

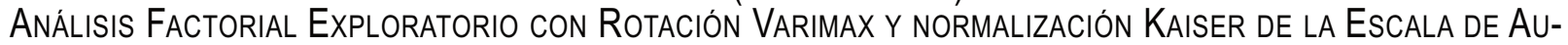
TOESTEREOTIPO ARGENTINO

\begin{tabular}{l|rr|}
\multicolumn{1}{c|}{ Itemes } & Factor 1 & Factor 2 \\
& & Factor 3 \\
\hline $\begin{array}{l}\text { Los argentinos somos: Vivos / Avivados } \\
\text { Los argentinos somos: No adaptativos / Rígidos }\end{array}$ & & .536 \\
Los argentinos somos: Charlatanes / Chamuyeros & & -.524 \\
Los argentinos somos: Poco expresivos / Inexpresivos \\
Los argentinos somos: Adaptativos / Flexibles & & .517 \\
\hline$\% \sigma^{2}$ explicada & & -.449 \\
$\% \sigma^{2}$ explicada total: $38.194 \%$ & 18.171 & .424 \\
\hline
\end{tabular}

TABLA 2

AnÁlisis de Conglomerado K-Medias de Perfiles

\begin{tabular}{|l|cc|}
\hline & \multicolumn{2}{|c|}{$\begin{array}{c}\text { Centros de Conglomerados Finales } \\
\text { Pesimista }\end{array}$} \\
\hline Argentino positivo & 3.44 & 2.72 \\
Argentino negativo & 2.77 & 3.26 \\
Argentino avivado & 4.34 & 3.77 \\
\hline
\end{tabular}




\section{REFERENCIAS BIBLIOGRÁFICAS}

Abrams, D. \& Hogg, A. (1990). An introduction to the social identity approach. En D. Abrams \& M.A. Hogg (Eds.), Social identity theory: Constructive and critical advances (pp. 1-9). Nueva York: Harvester Wheatsheaf.

Abrams, D. \& Hogg, M.A. (1988). Comments on the motivational status of self esteem in social identity and intergroup discrimination. European Journal of Social Psychology, 18, 317-334. http://dx.doi.org/10.1002/ejsp.2420180403

Alonso, D.R. \& Brussino, S.A. (2012). Legitimidad y percepciones generales de justicia de las autoridades legales de Córdoba (Argentina): Un abordaje desde las valoraciones de justicia procedimental [Legitimacy and general perceptions of judicial authorities from Córdoba (Argentina): An approach from procedural justice perceptions]. Interdisciplinaria, 29(2), 271-286.

Banchs, M.A., Cadenas, J.M., Domínguez, D. \& Montero, M. (1993). Identidad nacional: Permanencia y cambio [National identity: Permanence and change]. Revista Interamericana de Psicología, 27, 107-114.

Beramendi, B. \& Zubieta, E. (2013). La identidad nacional y relaciones interpersonales en una cultura donde la norma es la transgresión [National identity and interpersonal relationships in a culture where transgression is the norm]. Revista de Psicología Política, 13(26), 165-177.

Beramendi, M. \& Zubieta, E. (en prensa). La construcción y validación de la Escala de Percepción del Sistema Normativo [Construction and validation of the Normative System Perception Scale]. Revista Mexicana de Psicología.

D'adamo, O.J. \& García Beaudoux, V. (1995). El argentino feo [The ugly Argentinian]. Ciudad Autónoma de Buenos Aires: Losada.

Delfino, G.I. \& Zubieta, E.M. (2011). Valores y política: Análisis del perfil axiológico de los estudiantes universitarios de la ciudad de Buenos Aires (República Argentina) [Values and politic: Axiological profile analysis of college students in the city of Buenos Aires (Argentina)]. Interdisciplinaria, 28(1), 93-114.

Espinosa, A. (2003). Identidad social e identidad nacional en una muestra de triciclistas en Juliaca [Social and national identity in a sample of triciclistas in Juliaca]. Tesis de Licenciatura no publicada. Pontificia Universidad Católica del Perú, Lima.

Espinosa, A. (2011). Estudios sobre identidad nacional en el Perú y sus correlatos psicológicos, sociales y culturales [Studies about national identity in Peru and its psycologichal, social and cultural correlates]. Tesis Doctoral no publicada. Departamento de Psicología Social y Metodología de las Ciencias del Comportamiento. Universidad del País Vasco.

Espinosa, A., Beramendi, M. \& Zubieta, E. (2012). ¿Naciones saludables? Identidad nacional como fuente de bienestar subjetivo y social: Una sintesis meta-analitica de estudios en Argentina, México y Perú [Healthy nations? National identity as a source of subjective and social well-being: A meta-analytical synthesis of studies in Argentina, Mexico and Peru]. Trabajo presentado en el IV Congreso Latinoamericano de la ULAPSI, Montevideo.

Espinosa, A., Calderón, A., Burga, G. \& Güimac, J. (2007). Estereotipos, prejuicios y exclusión social en un país multiétnico: El caso peruano [Stereotypes, prejudice and social exclusion in a multiethnic country: The Peruvian case]. Revista de Psicología, 25(2), 295-338.

Espinosa, A. \& Tapia, G. (2011). Identidad nacional como fuente de bienestar subjetivo y social [National identity as a source of subjective and social well-being]. Boletín de Psicología, 102, 71-87.

Herencia, C. (1991). Identidad social en la dominación cultural y de clases en el Perú: Consecuencias para la identidad nacional [Social 
indentity in the cultutral and class dominance in Peru: Consequences for the national identity]. En M. Montero (Ed.), Acción y discurso. Problemas de psicología política en América Latina (pp. 117-153). Caracas: EDUVEN.

Herranz, J.K. \& Basabe, N. (1999). Identidad nacional, ideología política y memoria colectiva [National identity, political ideology and collective memory]. Psicología Política, 18, 31-47.

Hewstone, M., Rubin, M. \& Willis, H. (2002). Intergroup bias. Annual Review of Psychology, 53, 575-604.

Hogg, M.A. \& Ridgeway, C. (2003). Social identity: Sociological and social psychological perspectives. Social Psychology Quarterly, 66, 97-100.

Laca, F., Mejía, J.C. \& Yañez, C. (2010). Identidad mexicana e interés político: Predictores de bienestar social y anomia [Mexican identity and political interest: Social well-being and anomie predictors]. Acta Colombiana de Psicología, 20(2), 40-49.

Laca, F., Santana, H., Ochoa, Y. \& Mejía, J.C. (2011). Percepciones de bienestar social, anomia, interés e impotencia política en relación con las actitudes hacia la democracia [Social well-being perception, anomie, political interest and impotence in relation to the atittudes towards democracy]. Liberabit, 17(1), 7-18.

Latinobarómetro (2006). Análisis en línea: Igualdad ante la ley - derechos y obligaciones [Online analysis: Equality to the law - rights and obligations]. Recuperado el 15 de agosto de 2012 de http://www.latinobaro metro.org/lati no/LATAnalizeIndex.jsp

Latinobarómetro (2008). Análisis en línea: Igualdad ante la ley - derechos y obligaciones [Online analysis: Equality to the law - rights and obligations]. Recuperado el 15 de agosto de 2012 de http://www. latinobarometro.org/latino/LATAnalizeIndex.jsp

Latinobarómetro (2009). Análisis en línea: Igualdad ante la ley-derechos y obligaciones [On- line analysis: Equality to the law - rights and obligations]. Recuperado el 15 de agosto de $2012 \mathrm{de} \mathrm{http://www.latinobarometro.org/la-}$ tino/LATAnalizeIndex.jsp

Latinobarómetro (2010). Análisis en línea: Igualdad ante la ley - derechos y obligaciones [Online analysis: Equality to the law - rights and obligations]. Recuperado el 15 de agosto de 2012 de http://www.latinobarometro.org/latino/LATAnalizeIndex.jsp

Levin, S. \& Sidanius, J. (1999). Social dominance and social identity in the United States and Israel: Ingroup favoritism or outgroup derogation? Political Psychology, 20, 99-126. http://dx.doi.org/10.1111/0162-895X.00138

Luhtanen, R. \& Crocker, J. (1992). A collective self-esteem scale: Self evaluation of one's identity. Personality and Social Psychology Bulletin, 18, 302-318. http://x.doi.org/10.11 77/0146167292183006

Lyons, A.C., Cude, B., Lawrence, F.C. \& Gutter, M. (2005). Conducting research online: Challenges facing researchers in family and consumer sciences. Family and Consumer Sciences Research Journal, 33(4), 341-356. http://dx.doi.org/10.1177/1077727X04274116

Marín, G. \& Salazar, J. M. (1985). Determinants of hetero-and autostereotypes: Distance, level of contact, and socioeconomic development in seven nations. Journal of Cross-Cultural Psychology, 16, 403-422. http://dx.doi.org/ 10.1177/0022002185016004001

Mezulis, A.H., Abramson, L.Y., Hyde, J.S. \& Hankin, B.L. (2004). Is there a universal positivity bias in attributions? A meta-analytic review of individual, developmental and cultural differences in the self-serving attributional bias. Psychological Bulletin, 130(5), 711-747.

Montero, M. (1994). Altercentrismo y construcción de identidades negativas [Alter-centrism and the construction of the negative identities]. En D. Mato (Ed.), Teoría y política de la construcción de identidades y diferencias 
en América Latina y el Caribe (pp. 47-56). Caracas: UNESCO-Nueva Sociedad.

Montero, M. (1996). Identidad social negativa y crisis socioeconómica: Un estudio psicosocial [Negative social identity and socioeconomic crisis: A psychosocial study]. Revista Interamericana de Psicología, 30, 43-58.

Nigbur, D. \& Cinnirella, M. (2007). National identification, type and specificity of comparison and their effects on descriptions of national character. European Journal of Social Psychology, 37, 672-691. http://dx.doi.org/ 10.1002/ejsp.382

Pérez de León, P. (2007). Identidades, actitudes y estereotipos nacionales y supranacionales en una muestra uruguaya [Identities, attitudes and national and supranational stereotypes in a uruguayan sample]. Ciencias Psicológicas, 1 (1), 81-102.

Pérez, T. (1999). Nación, identidad nacional y otros mitos nacionalistas [Nation, national identity and other nationalist myths]. Oviedo: Nobel.

Pratto, F., Sidanius, J. \& Levin, S. (2006). Social dominance theory and the dynamics of intergroup relations: Taking stock and looking forward. European Review of Social Psychology, 17, 271-320. http://dx.doi.org/10.1080/1046 3280601055772

Pratto, J., Sidanius, J., Stallworth, L. \& Mallé, B. (1994). Social dominance orientation: A personality variable predicting social and political attitudes. Journal of Personality and Social Psychology, 67, 741-763. http://dx.doi.org/10. 1037/0022-3514.67.4.741

Romero, L.A. \& Bertoni, L.A. (1989). Movimientos migratorios en el Cono Sur, 1810-1930. En B. Leander (Ed.), Europa, Asia y África en América Latina y el Caribe [Europe, Asia and Africa to Latin America and the Caribbean], (pp. 160-216). México: Siglo XXI.

Roselli, N. (2000). Representación social de "los argentinos", "los españoles", "los latinoameri- canos" y "los europeos" en estudiantes universitarios argentinos [Social representation of "the Argentinian", "the Spaniards", "the Latinamericans" and "the Europeans" in undergraduates]. Revista Latinoamericana de Psicología, 32(1), 127- 160.

Rottenbacher, J.M. (2008). La valoración de la historia, la percepción del clima socio-emocional y su relación con la construcción de la identidad nacional en el Perú [History valoration, social and emotional atmosphere perception and it's relations with the construction of the national identity in Peru]. Tesis de Licenciatura no publicada, Pontificia Universidad Católica del Perú, Lima.

Rottenbacher, J.M. \& Espinosa, A. (2010). Identidad nacional y memoria histórica colectiva en el Perú. Un estudio exploratorio [National identity and historical collective memory in Peru. An exploratory study]. Revista de Psicología, 28(1), 147-174.

Rottenbacher, J.M. \& Schmitz, M. (2012). Conservadurismo político y tolerancia hacia comportamientos transgresores [Political conservatism and transgressive behaviour tolerance]. Psicología Política, 44, 31-56.

Salazar, J.M. \& Marín, G. (1977). National stereotypes as a function of conflict and territorial proximity: A Test of the Mirror Image Hypothesis. Journal of Social Psychology, 101, 13-19. http://dx.doi.org/10.1080/00224 545.1977.9923979

Salazar, J.M. \& Salazar, M.A. (1998). Estudios recientes acerca de identidades nacionales en América Latina [Recent studies about the national indetities in Latin America]. Psicología Política, 16, 75-93.

Salgado, C. (1999). ¿Quiénes somos los peruanos? Una perspectiva psicológica de la identidad nacional [Who are we the peruans? A psyhocological perspective of the national identity]. Trabajo no publicado. Lima: Escue- 
la Profesional de Psicología de la Universidad San Martín de Porres.

Sautú, R., Bonolio, P. \& Perugorría, I. (2004). Percepciones de corrupción y confianza en actores políticos y económicos [Perception of corruption and trust in political and economical figures]. En R. Sautú (Ed.), Catálogo de prácticas corruptas: Corrupción, confianza y democracia (pp. 139-166). Ciudad Autónoma de Buenos Aires: Lumiere.

Schmitt, M., Branscombe N.R. \& Kappen, D.N. (2003). Attitudes toward group-based inequality: Social dominance or social identity? British Journal of Social Psychology, 42(2), 161-186.

Sidanius, J. \& Pratto, F. (1999). Social dominance: An intergroup theory of social hierarchy and oppression. Cambridge: Cambridge University Press. http://dx.doi.org/10.1017/ CBO9781139175043

Smith, P.B., Giannini, M., Helkama, K., Maczynski, J. \& Stumpf, S. (2005). Positive auto-stereotyping and Self-construal as predictors of national identification. International Review of Social Psychology, 18, 6590.

Stanley, G. \& Glass, J. (1986). Métodos estadísticos [Statisical methods]. México: Prentice Hall.
Tajfel, H. (1982). Social psychology of intergroup relations. Annual Review of Psychology, 33, 1-39. http://dx.doi.org/10.1146/ann urev.ps.33.020182.000245

Tajfel, H. \& Turner, J. (1979). An integrative theory of intergroup conflict. En G. Austin \& S. Worchel (Eds), The social psychology of intergroup relations (pp. 33-47). Monterrey, CA: Brooks/Cole.

Vignoles, V.L. \& Moncaster, N. (2007). Identity motives and in-group favouritism: A new approach to individual differences in intergroup discrimination. British Journal of Social Psychology, 46, 91-113. http://dx.doi.org/10.13 48/014466605X85951

Vignoles, V.L., Regalia,C., Manzi, C., Golledge, J. \& Scabini, E. (2006). Beyond self-esteem: Influence of multiple motives on identity construction. Journal of Personality and Social Psychology, 90, 308-333. http://dx.doi.org/ 10.1037/0022-3514.90.2.308

Weber, J.A. \& Bradley, K.D. (2006). Strengths and weaknesses of conducting web-based surveys: A review of the literature. Trabajo presentado en el Mid-Western Educational Research Association Annual Meeting. Columbus, OH. Recuperado el 15 de agosto de 2012 de http:// www.uky.edu/ kdbrad2/WebbasedSurveys. pdf

Universidad de Buenos Aires (UBA) Ciudad Autónoma de Buenos Aires República Argentina

Fecha de recepción: 7 de diciembre de 2012 Fecha de aceptación: 12 de diciembre de 2013 
